\title{
Development and characterization of new phosphorus based flame retardant tetraglycidyl epoxy nanocomposites for aerospace application
}

\author{
K SHREE MEENAKSHI*, E PRADEEP JAYA SUDHAN and S ANANDA KUMAR \\ Department of Chemistry, Anna University, Chennai 600 025, India
}

MS received 28 January 2011

\begin{abstract}
A study was made in the present investigation on the development and characterization of triphenyl phosphine oxide based phosphorus tetraglycidyl epoxy nanocomposites denoted as ' $C$ ' and to find out its suitability for use in high performance applications. The synthesized resin was characterized by Fourier transform infrared spectra (FT-IR) and ${ }^{1} \mathrm{H},{ }^{13} \mathrm{C}$ nuclear magnetic resonance (NMR) spectra. Nanoclay and POSS-amine nanoreinforcements denoted as $\mathrm{N} 1$ and $\mathrm{N} 2$ were incorporated into the synthesized epoxy resin. Curing was done with diaminodiphenylmethane (DDM) and bis(3-aminophenyl) phenylphosphine oxide (BAPPO) curing agents denoted as $X$ and $Y$, respectively. Mechanical, thermal, flame retardant, water absorption behaviour and electrical properties of the epoxy nanocomposites were studied and the results are discussed.
\end{abstract}

Keywords. Epoxy resin; polyhedral oligomeric silsesquioxanes; phosphorus epoxy resin; thermal stability; flame retardancy; limiting oxygen index.

\section{Introduction}

Epoxy resins are used worldwide on a large scale for adhesive, laminating, coating and casting applications. They show several excellent characteristics: moisture, solvent and chemical resistance; superior electrical and mechanical properties; and good adhesion to many substrates. Their versatility has led to them being widely applied industrially. The chemistry and technological applications of the epoxy resins are very demanding and there are many new developments each year (Lee and Nevellie 1967; May et al 1988; Wong 1988; Cheng et al 1999; Ahmad et al 2005). However, their main disadvantage is that they are more flammable than similar thermosets since they have a reduced tendency to carbonize. After the ignition source has been removed they continue to burn on their own. In recent years, modification of epoxy resins has received increasing attention. In an attempt to improve their flame resistance, so-called intumescent systems are being currently developed to be environmentally friendly flame retardants, providing low smoke and toxicity exposure, as possible alternatives to traditional halogen based systems.

Phosphorus compounds are well established as components of flame retardant additives. Phosphorus containing flame retardants influence the reaction taking place in the condensed phase. Their effectivity depends on the chemical structure of the polymer and they are particularly effec-

\footnotetext{
*Author for correspondence (shreemeenakshik@gmail.com)
}

tive in materials with high oxygen content such as epoxies. The approaches of flame retardation of epoxy resins can be achieved by both additive type flame retardants and chemically bonding flame retardant groups onto the epoxy resin. However, flame retardants of the chemically bonding type are of interest recently because they exhibit several advantages over the additive type (Buckingham et al 1996; Cheng et al 2002; Jose Alcon et al 2003; Hussain et al 2004).

Polyhedral oligomeric silsesquioxanes (POSS) reagents are emerging as a new chemical technology for the nano-reinforced organic-inorganic hybrids. Typically POSS nanoparticle is a $3 \mathrm{D}$ cage like siloxane structure surrounded by eight organic ' $R$ ' groups $\left(R \mathrm{SiO}_{1.5}\right)$. These organic groups can be hydrocarbon species, which allow compatibilization and solubility of the particles into the polymer matrices or they can embody a large range of functional groups suitable for the preparation of nanohybrids by copolymerization or grafting. Incorporation of POSS into polymers like acrylics, styryls, epoxy and polyethylene has led to enhancements in thermal stability and mechanical properties, glass transition and degradation temperatures, oxygen permeability, reduced flammability and heat evolution as well as modified mechanical properties relative to conventional organic systems (Choi et al 2001; Baney et al 2005; Liu et al 2005; Huang et al 2009).

Polymer-clay nanocomposite $(\mathrm{PCN})$ is an area of polymer technology that has drawn considerable interest in recent years. The nanoscopic phase distribution as well as synergism between polymer and layered silicate results in additional properties such as flame retardation, enhanced barrier properties and ablation resistance. Layered silicates are 
proven to possess excellent barrier resistance against movement of water or gas molecules and other chemicals due to their high aspect ratio (Giannelis 1996; Kornmann et al 2002; Becker et al 2003; Gacitua 2005; Ipek Nakas and Kaynak 2009). Extensive research has been carried out on the preparation and exfoliation mechanisms of epoxy/layered silicate nanocomposites. It has been demonstrated by several researchers that both Young's modulus and fracture toughness improved with the incorporation of layered silicates.

An attempt has been made in the current investigation to synthesize triphenyl phosphine oxide based phosphorus tetraglycidyl epoxy nanocomposites. Curing of the synthesized nanocomposites is done using DDM and phosphorous containing curing agent (BAPPO). Mechanical study of the cured samples such as tensile, flexural and impact testing, thermal studies like thermogravimetric analysis (TGA), limiting oxygen index (LOI), water absorption and flame resistance of the epoxy resin along with the electrical properties are studied by modifying it with POSS-amine as nano reinforcement and organic modified clay as nano filler and interesting results obtained are discussed.

\section{Experimental}

\subsection{Materials}

All chemicals were of reagent grade and were used without further purification. 4,4'-diaminodiphenylmethane (DDM) was obtained from Huntsman, USA. Epichlorohydrin (ECH) and sodium hydroxide were obtained from SD Fine Chemicals, India. Triphenylphosphine oxide was obtained from Alfa-Aaser, Germany. Aminopropyltriethoxysilane ( $\gamma$-APS), 1,3,5,7,9,11,14 Hepta siloxane-endo-3,7,14 triol (POSStriol), stannous chloride and sulphuric acid were obtained from Merck (Germany). Tetrahydrofuran (THF) and benzene were obtained from Sisco Research laboratories, India. Nano clay was obtained from Nanocor, USA. Hydrochloric acid was obtained from Hi-pure, India.

\subsection{Synthesis of phosphorus tetraglycidyl epoxy resin}

2.2a Synthesis of phosphorus containing diamine [bis(3aminophenyl) phenyl phosphine oxide (BAPPO)]: Phosphorus diamine was synthesized according to the reported procedure (Liu et al 1997). Triphenyl phosphine oxide $(27.8 \mathrm{~g})$ was placed in a $500 \mathrm{ml}$ round bottom flask equipped with stirrer and nitrogen atmosphere and $200 \mathrm{ml}$ of $96 \%$ sulphuric acid was added to it. The reactant was dissolved and the reaction system was cooled to $-5^{\circ} \mathrm{C}$ with an ice/salt bath. A solution of $14.5 \mathrm{~g}$ of fuming nitric acid in $100 \mathrm{ml}$ of sulphuric acid was added drop wise over a period of $2 \mathrm{~h}$. The reaction system was kept at room temperature for another $8 \mathrm{~h}$. The reaction mixture was hydrolyzed by 2 litres of ice and the mixture was extracted with chloroform and washed with aqueous sodium bicarbonate solution until neutral $\mathrm{pH}$ was obtained. The solvent was removed. The solid residue was re-crystallized from absolute ethanol. Bis(3-nitrophenol) phenyl phosphine oxide (BNPPO) was obtained.

BNPPO was taken in a $1000 \mathrm{ml}$ round bottom flask with $180 \mathrm{~g}$ of anhydrous powder, tin (II) chloride. A solution of $200 \mathrm{ml}$ fuming hydrochloric acid in $400 \mathrm{ml}$ ethanol was introduced into the flask. The reaction mixture was stirred at room temperature for $5 \mathrm{~h}$. The solution was concentrated and then neutralized by $25 \%$ of aqueous sodium hydroxide solution. The obtained solution was extracted with chloroform. The organic layer was collected and concentrated under a reduced pressure. The obtained solid was recrystallized from dichloromethane to give a pure product of bis(3-aminophenyl) phenyl phosphine oxide (BAPPO). The synthesis is depicted in scheme 1 .
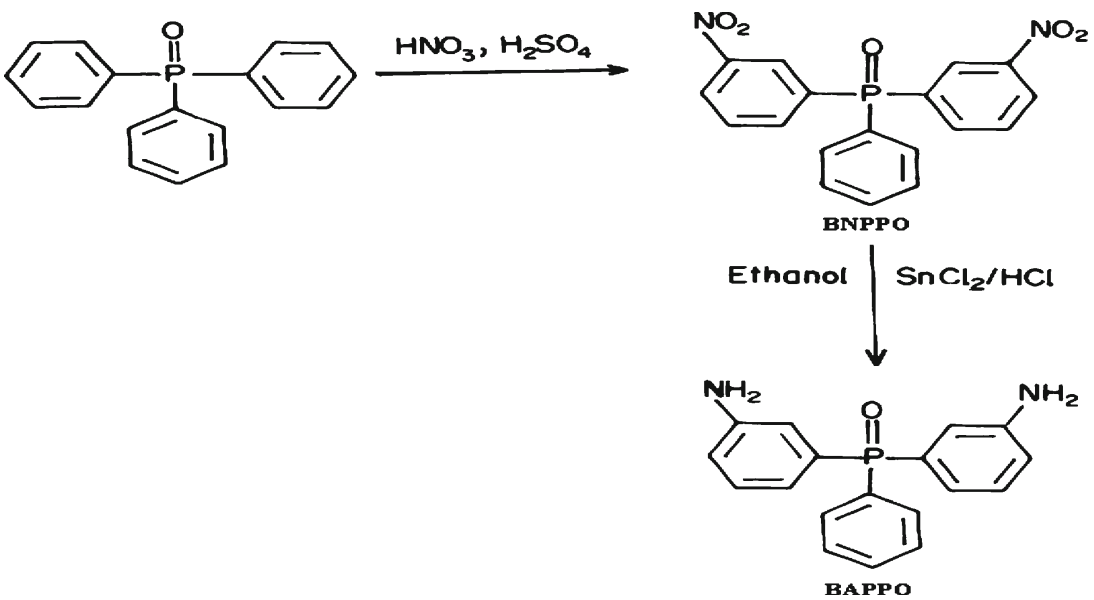

Scheme 1. Synthesis of phosphorus diamine. 
<smiles>Nc1cccc(P(=O)(c2ccccc2)c2cccc(C3CC3)c2)c1</smiles><smiles>O=P(c1ccccc1)(c1cccc(N(CC(O)CCl)CC(O)CCl)c1)c1cccc(N(CC(O)CCl)CC(O)CCl)c1</smiles><smiles>O=P(c1ccccc1)(c1cccc(N(CC2CO2)CC2CO2)c1)c1cccc(N(CC2CO2)CC2CO2)c1</smiles>

Scheme 2. Synthesis of phosphorus-containing tetraglycidyl epoxy resin (TG-P-1).

2.2b Synthesis of phosphorus-containing tetraglycidyl epoxy resin (TG-P-1): $576 \cdot 3 \mathrm{~g}(6 \cdot 25$ moles) of ECH was taken in a 1 litre three necked round bottom flask provided with a mechanical stirrer, nitrogen inlet tube and a water condenser. This was heated to $50^{\circ} \mathrm{C}$ in a water bath. Then $93.8 \mathrm{~g}$ (0.47 moles) of bis(3-aminophenyl) phenyl phosphine oxide was stirred continuously for $4 \mathrm{~h}$ at a temperature range of $50-55^{\circ} \mathrm{C}$ under nitrogen atmosphere. Chlorohydrin, the intermediate product, was formed and excess ECH used was distilled off under vacuum. Then $113.7 \mathrm{~g}(2.84$ moles) of $40 \% \mathrm{NaOH}$ solution was added drop wise for $1 \mathrm{~h}$ at $54^{\circ} \mathrm{C}$. Then the reaction was continued at this temperature for another $1 \mathrm{~h}$. Then $250 \mathrm{ml}$ benzene was added to the mixture and stirred for $15 \mathrm{~min}$ to get two layers. The aqueous layer with $\mathrm{NaCl}$ by product was formed. While organic layer was purified by washing several times with water. Then benzene was evaporated to get light brown coloured liquid epoxy product with $65 \%$ yield. The synthesis is depicted in scheme 2.

\subsection{Synthesis of amine functionalized POSS $\left(\mathrm{POSS}-\mathrm{NH}_{2}\right)$}

Synthesis of POSS- $\mathrm{NH}_{2}$ was carried out as per the procedure shown in scheme 3. Stoichiometric amount of POSStriol (11.84 g) dissolved in dry toluene was mixed with
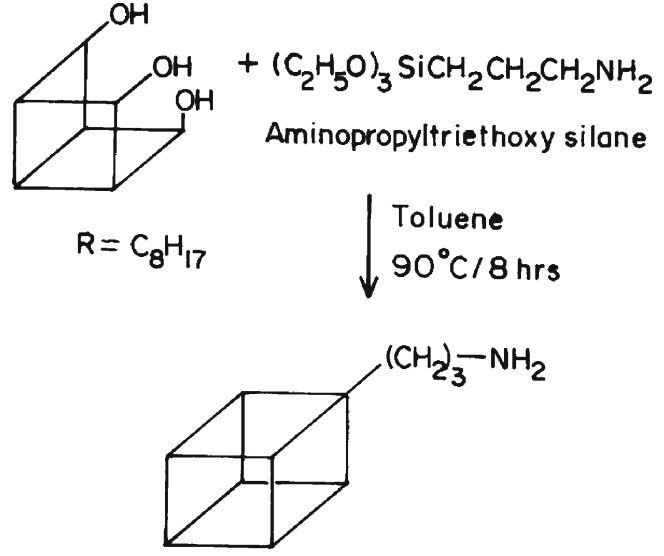

Scheme 3. Synthesis of amine-functionalized POSS (POSS$\mathrm{NH}_{2}$ ).

Table 1. Fabrication of neat resin laminates.

\begin{tabular}{lccc}
\hline $\begin{array}{l}\text { Type of } \\
\text { epoxy }\end{array}$ & $\begin{array}{c}\text { Matrix } \\
\text { name }\end{array}$ & Nanoreinforcement & $\begin{array}{r}\text { Curing } \\
\text { agent }\end{array}$ \\
\hline TG-P-1 & CX & - & $X$ \\
& CXN1 & N1 & $X$ \\
& CXN2 & N2 & $X$ \\
& CY & - & $Y$ \\
& CYN1 & N1 & $Y$ \\
& CYN2 & N2 & $Y$ \\
\hline
\end{tabular}

aminopropyltriethoxysilane $(1.63 \mathrm{~g})$ in a $50 \mathrm{ml}$ round bottomed flask and refluxed for $8 \mathrm{~h}$ at $90^{\circ} \mathrm{C}$. The filtrate was then subjected to solvent evaporation to obtain the desired product.

\subsection{Fabrication of neat resin laminates}

In order to study properties of the epoxy resins, neat resin laminates were prepared by curing the synthesized tetraglycidyl epoxy resins by DDM and BAPPO curing agents as shown in table 1 . For the fabrication of nanocomposites, the nanoclay (Nanocor $1.30 \mathrm{E}$ ) was dried for $24 \mathrm{~h}$ at $50^{\circ} \mathrm{C}$ under vacuum. The epoxy resin was mixed mechanically in a reaction vessel with the nanoclay at $50^{\circ} \mathrm{C}$ for $3 \mathrm{~h}$. Then it was further mixed in an ultrasonic bath for $30 \mathrm{~min}$ to disperse the clay in the resin. Later the mixture was cooled to room temperature for $30 \mathrm{~min}$. The curing agent was then added. After mixing mechanically for $10 \mathrm{~min}$, the mixture was degassed by a vacuum pump to remove the air bubbles and poured into moulds. The nanocomposites were cured for $1 \mathrm{~h}$ at $120^{\circ} \mathrm{C}$ and post-cured for $2 \mathrm{~h}$ at $200^{\circ} \mathrm{C}$. After that the resin plaque was cooled to room temperature naturally. The nano-reinforced POSS amine was mixed in stoichiometric amounts with the epoxy resin and then cured. 


\section{Characterization}

FT-IR spectra were recorded using Perkin-Elmer 781 infrared spectrometer with $\mathrm{KBr}$ pellets for solid samples. NMR spectra were recorded on a Bruker $300 \mathrm{MHz}$ NMR spectrometer with dimethylsulfoxide- $d 6$ (DMSO- $d 6$ ) as solvent. Tensile properties are studied as per ASTM-D3039 using universal testing machine model (HTE-S series-H50K-S model Hounsfield test equipments Ltd., UK). Flexural properties are studied as per ASTM D790 using Hinstron testing machine. Fracture test was performed on a (HTE - Series H50K -S model, Hounsfield test equipments Ltd. UK) hydraulic testing machine according to ASTM D 5045-99. Thermogravimetric analysis (TGA) was carried out using Thermal Analyst 2000 (TA instruments USA) at a heating rate of $10^{\circ} \mathrm{C} / \mathrm{min}$ in an inert atmosphere.

\section{Results and discussion}

\subsection{Spectral analysis}

FT-IR spectrum of BAPPO is depicted in figure 1. The characteristic peaks appear at $3499 \mathrm{~cm}^{-1}$ and $3393 \mathrm{~cm}^{-1}$ which confirm the presence $\mathrm{Ph}-\mathrm{NH}_{2}$ group. The peak appearing at $1277 \mathrm{~cm}^{-1}$ confirms the presence of $\mathrm{P}=\mathrm{O}$ group. The absorption band at $1498 \mathrm{~cm}^{-1}$ is attributed to the $\mathrm{P}-\mathrm{Ph}$ stretching. The ${ }^{1} \mathrm{H}$ NMR spectrum of BAPPO is shown in figure 2. The resonance signal at $3.6 \mathrm{ppm}$ confirms the presence of $-\mathrm{NH}_{2}$ group. The signal at $6 \cdot 1-7.8 \mathrm{ppm}$ may be due to the aromatic amine proton. The FT-IR spectrum of phosphorus tetraglycidyl epoxy is shown in figure 3 . The peaks at $1596 \mathrm{~cm}^{-1}$ and $1488 \mathrm{~cm}^{-1}$ represent the absorption of the aromatic group. Absorptions arising because of $\mathrm{P}=\mathrm{O}$ is seen at $1154 \mathrm{~cm}^{-1}$. The characteristic band of the oxirane ring was observed at $890-910 \mathrm{~cm}^{-1}$. The band at $1323 \mathrm{~cm}^{-1}$ corresponds to $-\mathrm{P}-\mathrm{C}_{6} \mathrm{H}_{5}$ group.

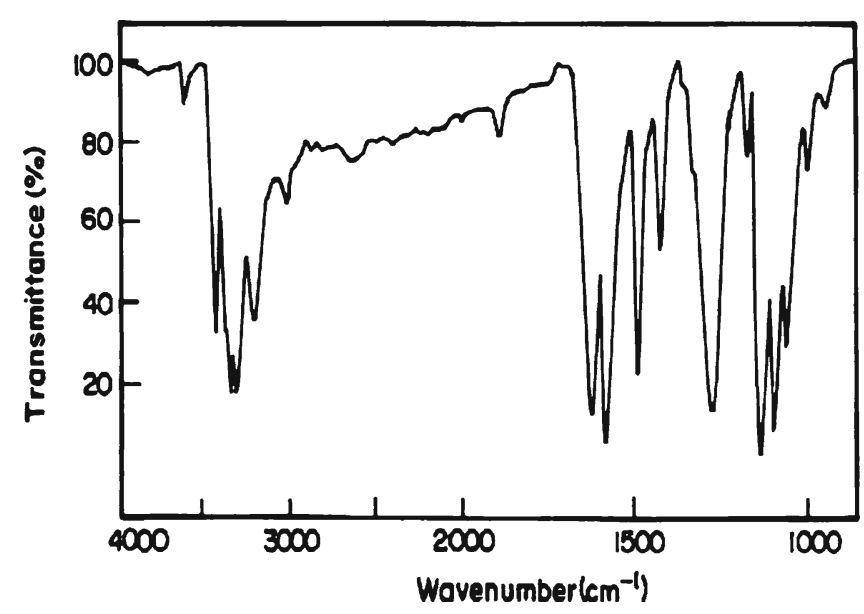

Figure 1. FTIR spectra of BAPPO.

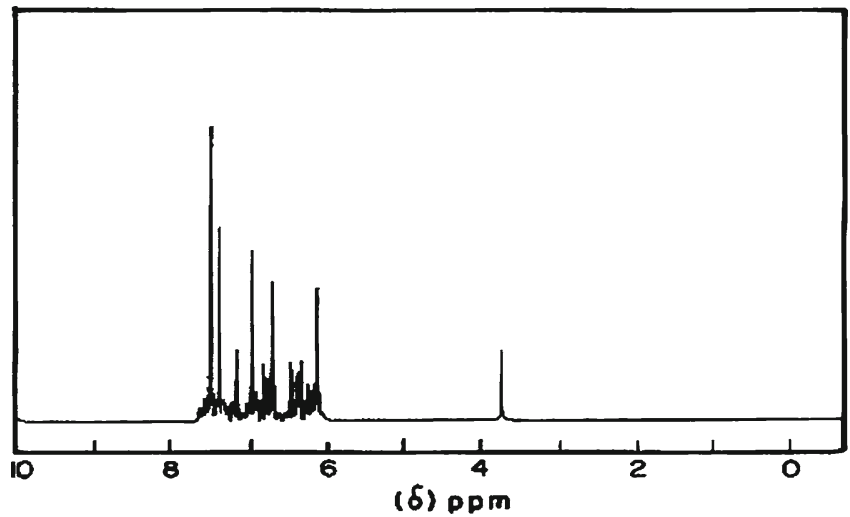

Figure 2. ${ }^{1} \mathrm{H}-\mathrm{NMR}$ spectra of BAPPO.

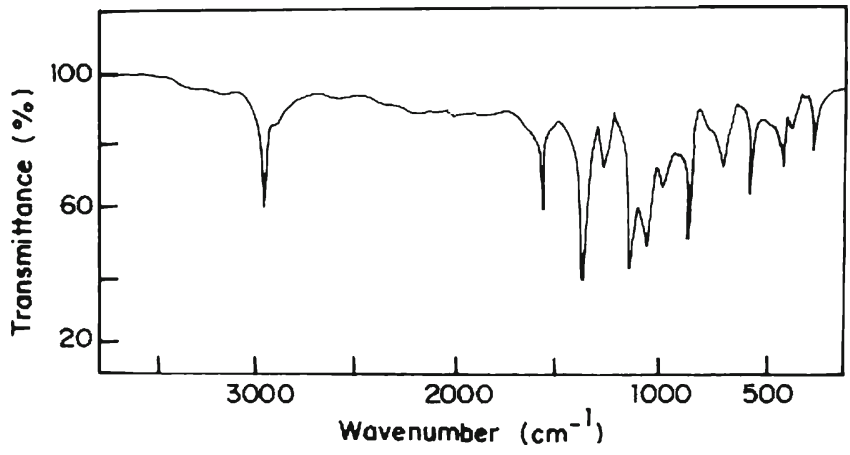

Figure 3. FTIR spectra of TG-P-1.

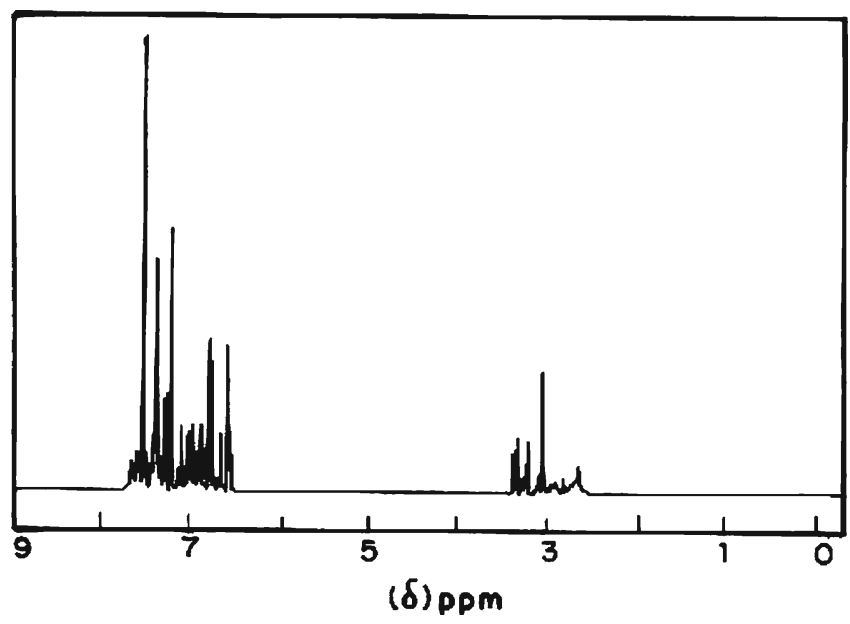

Figure 4. $\quad{ }^{1} \mathrm{H}-\mathrm{NMR}$ spectra of TG-P-1. 


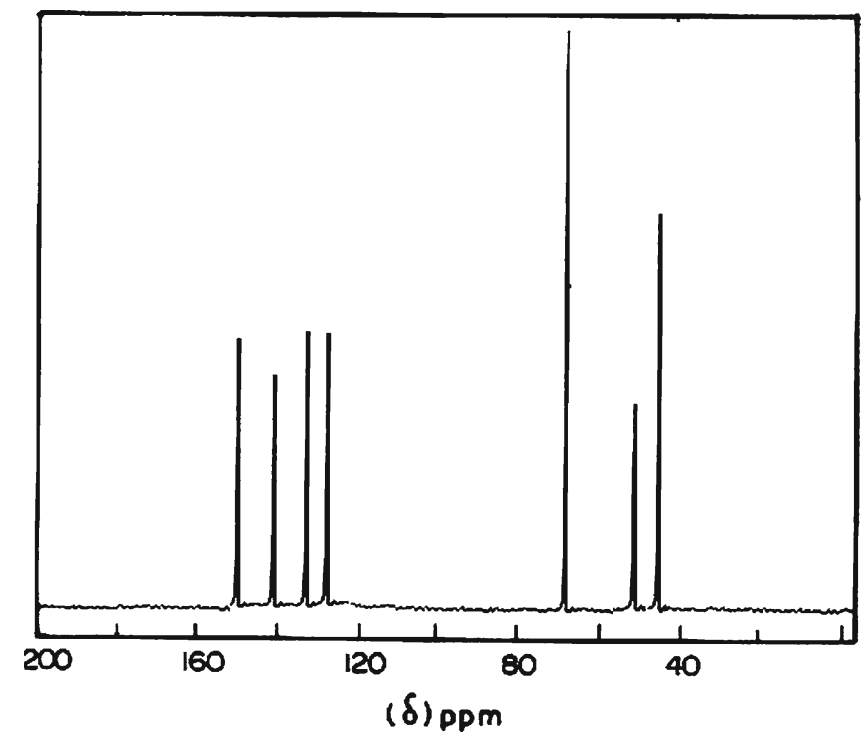

Figure 5. ${ }^{13} \mathrm{C}-\mathrm{NMR}$ spectra of TG-P-1.

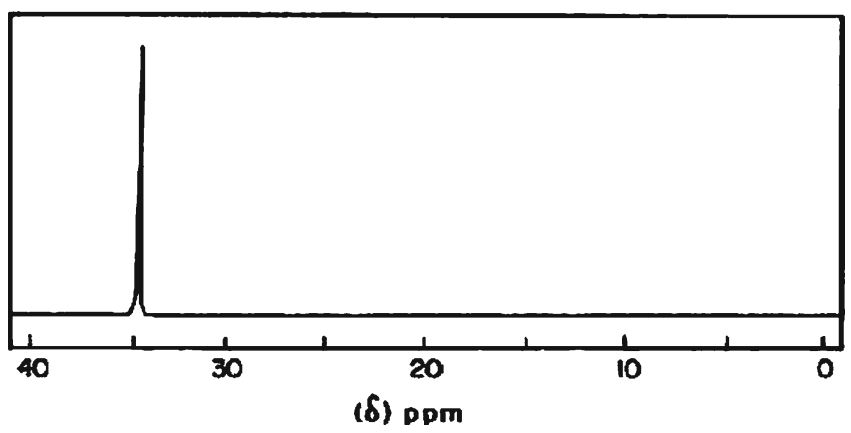

Figure 6. ${ }^{31} \mathrm{P}-\mathrm{NMR}$ spectra of TG-P-1.

The ${ }^{1} \mathrm{H}$ NMR spectrum of phosphorus tetraglycidyl epoxy is shown in figure 4 . The signal at $6.5-7.5 \mathrm{ppm}$ may be due to the aromatic proton, $\delta=2.69-3.8 \mathrm{ppm}$ (oxirane protons and methylene protons adjacent to oxirane). The ${ }^{13} \mathrm{C}$ NMR spectrum of phosphorus tetraglycidyl epoxy is shown in figure 5. The signal at about $50 \mathrm{ppm}$ may be due to the presence of - $\mathrm{CH}-$ epoxy group. The peak at $44 \mathrm{ppm}$ may be due to the $-\mathrm{OCH}_{2}-$ epoxy group. The signal at about $75 \mathrm{ppm}$ may be due to the presence of $-\mathrm{N}-\mathrm{CH}_{2}-$ carbons. The signal from $120-140 \mathrm{ppm}$ is due to the aromatic carbons. The single signal in the ${ }^{31} \mathrm{P}$ spectra at around $35 \mathrm{ppm}$ as seen in figure 6 confirms the structure of phosphorus tetraglycidyl epoxy.

The FT-IR spectrum of POSS- $\mathrm{NH}_{2}$ is depicted in figure 7. The peaks corresponding to $-\mathrm{NH}_{2}$ and $-\mathrm{CH}_{2}$ group of POSS- $\mathrm{NH}_{2}$ appear at $3300 \mathrm{~cm}^{-1}$ and $2900 \mathrm{~cm}^{-1}$, respectively. Furthermore, absorption peaks appeared at 1200 $1300 \mathrm{~cm}^{-1}$ and $1500 \mathrm{~cm}^{-1}$ which confirmed the presence of $\mathrm{Si}-\mathrm{O}-\mathrm{Si}$ linkage and $-\mathrm{Si}-\mathrm{CH}_{2}$, respectively. The ${ }^{1} \mathrm{H}$ NMR spectrum of POSS amine is shown in figure 8 . The signal at $0.64 \mathrm{ppm}$ may be due to the $\mathrm{Si}-\mathrm{CH}_{2}$ protons. The signal at $1.4 \mathrm{ppm}$ corresponds to $-\mathrm{C}-\mathrm{NH}_{2}$ protons.

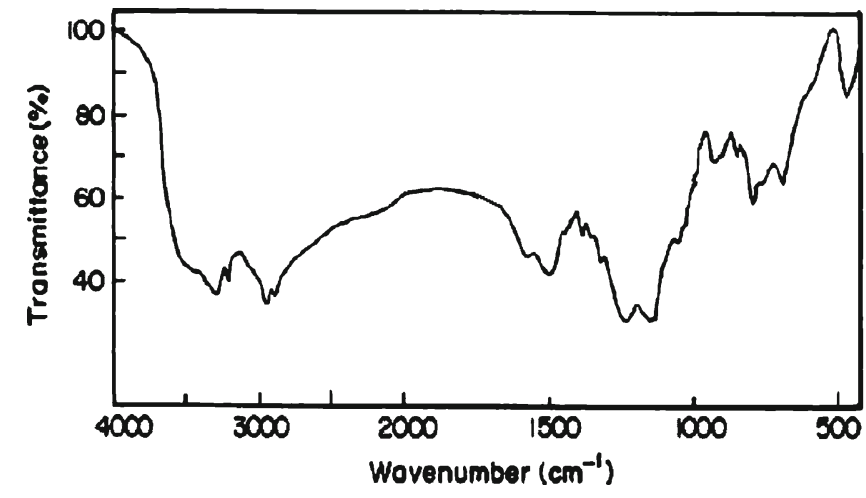

Figure 7. FTIR spectra of POSS-amine.

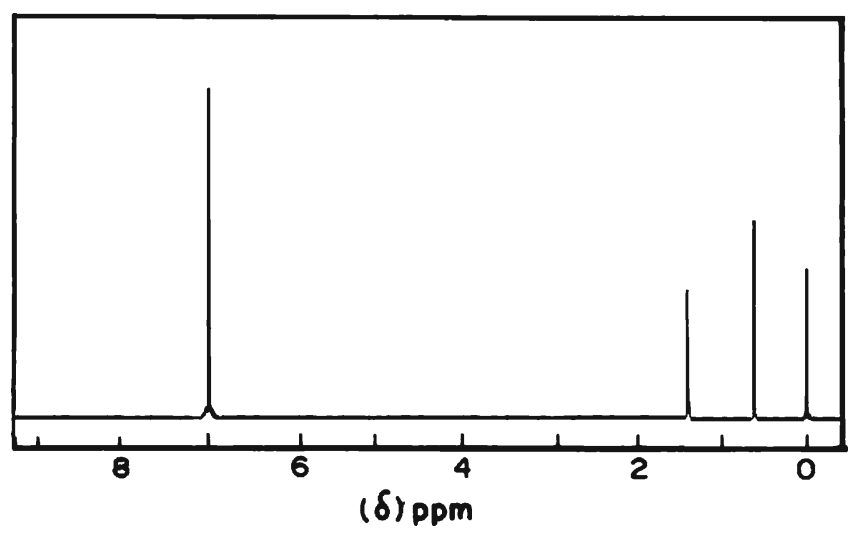

Figure 8. ${ }^{1} \mathrm{H}-\mathrm{NMR}$ spectra of POSS-amine.

\subsection{Mechanical characteristics}

4.2a Tensile, flexural and impact strength: The data resulting from the mechanical studies are given in table 2 . From the data, it is found that the incorporation of both nano clay (Nanomer 1.30E) and POSS amine into the virgin epoxy results in increase in tensile and flexural strengths. The values of the tensile and flexural modulus also increased. The reason for the increase in tensile strength and modulus may be due to the uniform dispersion of the nanoparticles of the layered silicates in the matrix of the polymer. The small size of POSS restricts the mobility of the polymer chain and thereby increases the modulus, and good interfacial adhesion between the POSS and the matrix also contributed to the reinforcement (McIntyre et al 2005). A lowering of the impact strength was observed which could be due to the restricted chain mobility due to the increase in cross linking and reduction in free volume. The impact values are found to be high for TG-phosphorus nanocomposites, which may be again due to the flexible nature of $\mathrm{Si}-\mathrm{O}-\mathrm{Si}$ linkage (Liu et al 2007). 
Table 2. Data resulting from the mechanical studies.

\begin{tabular}{lccccc}
\hline Resin system & $\begin{array}{c}\text { Tensile } \\
\text { strength } \\
(\mathrm{MPa})\end{array}$ & $\begin{array}{c}\text { Tensile } \\
\text { modulus } \\
(\mathrm{GPa})\end{array}$ & $\begin{array}{c}\text { Flexural } \\
\text { strength } \\
(\mathrm{MPa})\end{array}$ & $\begin{array}{c}\text { Flexural } \\
\text { modulus } \\
(\mathrm{GPa})\end{array}$ & $\begin{array}{c}\text { Impact } \\
\text { strength } \\
\left(\mathrm{kJ} / \mathrm{m}^{2}\right)\end{array}$ \\
\hline CX & 82 & 6.55 & 140 & 4.64 & 38 \\
CXN1 & 89 & 6.89 & 147 & 4.77 & 35 \\
CXN2 & 97 & 6.98 & 154 & 4.83 & 31 \\
CY & 88 & 6.88 & 145 & 4.69 & 36 \\
CYN1 & 95 & 6.93 & 152 & 4.81 & 33 \\
CYN2 & 102 & 7.10 & 160 & 4.90 & 30 \\
\hline
\end{tabular}

\subsection{Thermogravimetric analysis (TGA)}

The thermal analyses of the resins were studied by thermogravimetric analysis and from the data the thermal degradation temperature of the resins could be found. The char yield and LOI of the resins were found by TGA. The data obtained from the TGA studies is shown in table 3 and figures 9 and 10. It was observed that the systems cured with BAPPO performed better than the systems cured with DDM. The char yield and LOI were higher for the BAPPO systems as seen from table 3.

For example, the initial decomposition temperature of the CX system was $205^{\circ} \mathrm{C}$. The Y cured systems (CY, CYN1 and CYN2) showed a similar double decomposition pattern. In contrast to this observation, the $\mathrm{X}$ cured resin systems (CX, CXN1 and CXN2) showed a single decomposition pattern. The initial degradation temperature of the $Y$ cured system was lower than that of the $X$ cured systems, which was due to the decomposition of $\mathrm{P}-\mathrm{O}-\mathrm{C}$ bond at lower temperature. It was interesting to note that the char yield and LOI of the tetraglycidyl systems cured with $Y$ were found to be higher than those of $X$ cured systems. For example as seen from table 3, the char yield of CX was 49\% and LOI was 40, whereas the char yield of CY was 57\% (figure 10) and LOI was $43 \%$. The higher char yield observed for $Y$ cured systems may be due to the formation of a protective char layer formed as a result of the degradation that occurred at lower temperature, thereby protecting the underlying matrix from further degradation.

The addition of nanoreinforcements produced a significant improvement on the thermal stability, char yield and

Table 3. Data resulting from TGA analysis.

\begin{tabular}{lccc}
\hline Resin system & $\begin{array}{c}\text { Initial decomposition } \\
\text { temperature }\left({ }^{\circ} \mathrm{C}\right)\end{array}$ & $\begin{array}{c}\text { Char yield } \\
(\%)\end{array}$ & LOI \\
\hline CX & 205 & 49 & 40 \\
CXN1 & 210 & 52 & 41 \\
CXN2 & 230 & 55 & 42 \\
CY & 195 & 57 & 43 \\
CYN1 & 205 & 60 & 44 \\
CYN2 & 225 & 63 & 45 \\
\hline
\end{tabular}

LOI of all tetra functional epoxy resin systems. For example, the initial decomposition temperature of CX system was $205^{\circ} \mathrm{C}$ while the initial decomposition temperatures of CXN1 and CXN2 systems were significantly improved to $210^{\circ} \mathrm{C}$ and $230^{\circ} \mathrm{C}$, respectively as seen from table 3 and figure 9 . Similarly the char yield for the CX system was $49 \%$ and that for the CXN1 and CXN2 systems was 52\% and 55\%, respectively leading to an enhanced LOI values. Incorporation of POSS-amine significantly enhanced the thermal stability of the epoxy resin due to the formation of an inert silica layer on the surface of materials when decomposition takes place and prevented further oxidation of the inner part of the epoxy matrices. POSS molecules having silica like $\mathrm{Si}-\mathrm{O}-\mathrm{Si}$ structure, led to higher inorganic components in the cured materials resulting in higher char yields (Zhang et al 2007). This $\mathrm{Si}-\mathrm{O}-\mathrm{Si}$ linkage prevented the underlying polymeric matrix from further degradation. On the other hand, the clay nanolayers acted as barriers by preventing the evolution of volatile degradation products from the epoxy matrices (Leszcynska et al 2005). Between the two nanoreinforcements, the best results of thermal stability and flame retardancy were given by POSS-amine reinforced system, viz. CYN2.

\subsection{Flame and water resistance}

4.4a Flame retardant studies -UL -94 V Test (V-0, V-1, $V-2$ ): The data resulting from the flame retardant studies of the samples of phosphorus tetra epoxies and their nanocomposites are shown in table 4 . The flame retardancy enhances on incorporation of both POSS (Ni et al 2004) and clay (Kornmann et al 2002). This may be due to the char forming nature of clay and low surface energy of $\mathrm{Si}-\mathrm{O}-\mathrm{Si}$ present in POSS, which migrates to the surface and protect the underlying matrix. The tetraglycidyl phosphorus nanocomposites gave a better performance in flame test due to the high thermal stability of Si-O-Si linkage of nano-clay and POSS nano-reinforcement.

4.4b Water absorption studies (ASTM D570): The data resulting from the water absorption studies of the samples of tetra epoxy and the corresponding nanocomposites are 
Table 4. Flame retardant studies of TG-P-1 resin and nanocomposites.

\begin{tabular}{|c|c|c|c|c|c|c|}
\hline \multirow[b]{3}{*}{ Systems } & \multicolumn{6}{|c|}{ Flame test rating } \\
\hline & \multicolumn{2}{|c|}{ Pure system } & \multicolumn{2}{|c|}{ POSS amine system } & \multicolumn{2}{|c|}{ Clay system } \\
\hline & DDM & BAPPO & DDM & BAPPO & DDM & BAPPO \\
\hline TG-P-1 & V0 & V0 & V0 & V0 & V0 & V0 \\
\hline
\end{tabular}

Table 5. Water absorption studies of TG-P-1 resin and nanocomposites.

\begin{tabular}{|c|c|c|c|c|c|c|}
\hline \multirow[b]{3}{*}{ Systems } & \multicolumn{6}{|c|}{$\%$ of water absorption } \\
\hline & \multicolumn{2}{|c|}{ Pure system } & \multicolumn{2}{|c|}{ Clay system } & \multicolumn{2}{|c|}{ POSS amine system } \\
\hline & DDM & BAPPO & DDM & BAPPO & DDM & BAPPO \\
\hline TG-P-1 & 0.057 & 0.050 & 0.043 & 0.035 & 0.025 & 0.017 \\
\hline
\end{tabular}

Table 6. Data resulting from electrical studies.

\begin{tabular}{lccccc}
\hline Systems & $\begin{array}{c}\text { Dielectric } \\
\text { strength } \\
(\mathrm{kV} / \mathrm{mm})\end{array}$ & $\begin{array}{c}\text { Surface } \\
\text { resistivity } \\
(\mathrm{Ohm})\end{array}$ & $\begin{array}{c}\text { Volume } \\
\text { resistivity } \\
(\mathrm{Ohm})\end{array}$ & $\begin{array}{c}\text { Arc } \\
\text { resistance } \\
(\text { seconds })\end{array}$ & $\begin{array}{c}\text { Comparitive } \\
\text { tracking } \\
\text { index (Volts) }\end{array}$ \\
\hline $\mathrm{CX}$ & 21.2 & $3.66 \times 10^{13}$ & $>10^{14}$ & 113 & $>600$ \\
$\mathrm{CXN1}$ & 23.4 & $5.89 \times 10^{13}$ & $>10^{14}$ & 123 & $>600$ \\
$\mathrm{CXN} 2$ & 25.5 & $2.21 \times 10^{14}$ & $>10^{14}$ & 133 & $>600$ \\
CY & 22.3 & $4.26 \times 10^{13}$ & $>10^{14}$ & 121 & $>600$ \\
CYN1 & 24.8 & $1.68 \times 10^{14}$ & $>10^{14}$ & 130 & $>600$ \\
CYN2 & 26.7 & $3.63 \times 10^{14}$ & $>10^{14}$ & 142 & $>600$ \\
\hline
\end{tabular}

shown in table 5. The water absorption tendency decreases on addition of both POSS (Haque et al 2003) and clay (Liu et al 2005), which is due to the hydrophobic and partial ionic nature of $\mathrm{Si}-\mathrm{O}-\mathrm{Si}$ linkage. The TG-P-1 nanocomposites gave a better performance due to the hydrophobic nature of $\mathrm{Si}-\mathrm{O}-\mathrm{Si}$ linkage of nano-clay and POSS nanoreinforcement.

\subsection{Discussion on electrical properties}

The results of the various electrical studies are shown in table 6 .

As seen from table 6 , the dielectric strength of the neat CX system was $21.2 \mathrm{kV} / \mathrm{mm}$, whereas the dielectric values of CY system was enhanced to $22.3 \mathrm{kV} / \mathrm{mm}$. The Y cured systems were found to show better electrical properties than the $\mathrm{X}$ cured systems. This could be due to the presence of rigid aromatic groups of $\mathrm{Y}$ which might have enhanced the hydrophobic behaviour and may also be due to the formation of insulative char layer that contributed to better electrical properties. The incorporation of nanoreinforcements (nanoclay and POSS-amine) was found to enhance the electrical properties significantly. The nano-reinforced epoxy composites yielded breakdown strength values as high as that of the base resin indicating their insulative behaviour required for electrical application. For example, surface resistivity of CX system was $3.66 \times 10^{13} \mathrm{ohm}$ whereas for the system $\mathrm{CXN1}$ and $\mathrm{CXN} 2$, the surface resistivity was enhanced to $5.89 \times 10^{13} \mathrm{ohm}$ and $2.21 \times 10^{14} \mathrm{ohm}$, respectively. Similar observation was noticed for the dielectric strength and arc resistance measurements of nanocomposites. This could possibly be due to the presence of the siloxane linkages present in the nanoreinforcements that may be responsible for the enhanced insulative properties.

\section{Conclusions}

The present work deals with the development and characterization of high functionality epoxy resin, viz. DOPO based phosphorus tetraglycidyl epoxy resin. This epoxy resin was cured with DDM and BAPPO, respectively with and without the incorporation of nanoclay (Nanomer $1.30 \mathrm{E}$ ) and nano-reinforcement (POSS amine) to get matrix materials to be utilized for aerospace applications. The developed materials were characterized by spectral studies to ascertain 
their structures. The polymerization of these resins was carried out in the presence and absence of nanoclay and nanoreinforcement to get the nanocomposites. The thermal studies of these materials were carried out by means of TGA and the results obtained from this study clearly indicate that BAPPO (Y) cured tetra epoxies, irrespective of nanoclay and nano-reinforcement showed better char yield and LOI values in spite of their degradation at lower temperature. The incorporation of nanoclay and nano-reinforcement to the epoxy improved the IDT, char yield and LOI values.

The Y cured systems were found to show better electrical properties than that of $\mathrm{X}$ cured systems. This could be due to the presence of rigid aromatic groups which enhanced the hydrophobic behaviour and also due to the formation of insulative char layer that contributed to improved electrical properties. The addition of nano-reinforcements (nanoclay and POSS-amine) was found to significantly enhance the electrical properties. For example, the arc resistance value of the neat system CX was found to be $113 \mathrm{~s}$, while for the nanocomposites CXN1 and CXN2 the values were enhanced to $123 \mathrm{~s}$ and $133 \mathrm{~s}$, respectively. This could be accounted due to the presence of the siloxane linkages present in the nano-reinforcements that enhanced the insulative properties.

From the data obtained from different studies, it can be concluded that the novel organic-inorganic nano hybrid composites synthesized in the present study, having improved mechanical, thermal properties being self extinguishable, heat resistant at the same time with excellent dielectric properties, can very well be used for automotive, electronics and advanced aerospace application for improved performance and longevity than the materials that are currently in use.

\section{Acknowledgements}

The authors are grateful to the Council for Scientific and Industrial Research, Govt. of India and the Department of Science and Technology, Govt. of India, for having provided financial assistance to carry out this work and are also thankful to Anna University, Chennai, for providing necessary facilities for this work.

\section{References}

Ahmad S, Gupta A P, Sharmin E, Alam M and Pandey S K 2005 Prog. Org. Coat. $\mathbf{5 4} 248$

Baney R H, Itoh M, Sakakibara A and Suzuki T 2005 Chem. Rev. 951409

Becker O, Simon G P, Varley R J and Halley P J 2003 Polym. Eng. Sci. 43850

Buckingham M R, Lindsay A J, Stevenson D E, Muller G, Morel E, Costes B and Henry Y 1996 Polym. Degrad. Stab. 54311

Cheng K C, Lai K C and Chiu W J 1999 J. Appl. Polym. Sci. 71721

Cheng K C, Yu S Y and Chiu W Y 2002 J. Appl. Polym. Sci. 832741

Choi J, Harcup J, Yee A F, Zhu Q and Laine R M 2001 J. Am. Chem. Soc. 12311420

Gacitua W 2005 Maderas. Cienc. Tecnol. 7159

Giannelis E P 1996 Adv. Mater. 8290

Haque A, Shamsuzzoha M, Hussain F and Dean D 2003 J. Compos. Mater. $\mathbf{3 7} 1821$

Huang J M, Huang H J, Wang Y X, Chen W Y and Chang F C 2009 J. Polym. Sci. Part B: Polym. Phys. 471927

Hussain M, Varley R J, Mathys Z, Cheng Y B and Simon G P 2004 Appl. Polym. Sci. 911233

Ipek Nakas G and Kaynak C 2009 Polym. Compos. 30357

Jose Alcon M, Gemma Ribera, Marina Galia and Virginia Cadiz 2003 Polymer $\mathbf{4 4} 7291$

Kornmann X, Thomann R, Mulhaupt R, Finter J and Berglund L A 2002 Polym. Eng. Sci. 421815

Lee H and Nevellie K 1967 Handbook of epoxy resins (New York: McGraw-hill)

Leszczynska A, Njuguna J, Pielichowski K and Banerjee J R 2005 Thermochim. Acta $\mathbf{4 5 3} 75$

Liu H, Zheng S and Nie K 2005 Macromol. 385088

Liu W, Hsiue G H, Lee R H and Chiu Y S 1997 J. Appl. Polym. Sci. 63895

Liu W, Varley R J and Simon G P 2007 Polymer 482345

Liu W P, Hoa S V and Pugh M D 2005 Compos. Sci. Technol. 65 2364

May C A 1988 Epoxy resins-chemistry and technology (New York: Marcel Dekker)

McIntyre S, Kaltzakorta I, Liggat J J, Pethrick R A and Rhoney I 2005 Ind. Eng. Chem. Res. 448573

Ni Y, Zheng S and Nie K 2004 Polymer 455557

Wong C P 1988 Encyclopedia of polymer science and engineering (New York: Wiley)

Zhang Z, Liang G and Wang X 2007 Polym. Bull. 581013 